\title{
LA ECOLOGÍA EN COLOMBIA: RELACIÓN, IMPACTOS Y ATENUACIÓN CON LOS SECTORES MINERO, ENERGÉTICO Y PORTUARIO
}

\section{ECOLOGY IN COLOMBIA. RELATION, MITIGATION AND IMPACT ON THE ENERGY, MINING, AND PORT SECTOR}

\author{
Jorge Enrique Pineda Patiño*
}

Pineda P. Jorge E. Sophia N 7 - 2011. ISSN: 194-8932 Págs. 77-91.

Recepción: Junio 15 de 2011

Aceptación: Agosto 3 de 2011

\section{RESUMEN}

En primer lugar, etimológicamente ecología significa, el estudio del lugar donde se habita; de acuerdo con Haeckel (1870), ecología, indica el cuerpo de conocimiento relativo a la economía de la naturaleza, con otros términos, la investigación de las relaciones totales del animal con su ambiente tanto orgánico como inorgánico, las cuales incluyen sobre todo su relación amistosa y hostil con aquellos animales y plantas con los cuales entra directa o indirectamente en contacto. Más brevemente, la Ecología es el estudio de todas las interrelaciones complejas, a las que se refería Darwin, así como las condiciones de lucha por la existencia; siendo así, la ciencia de la ecología, a menudo considerada equivocadamente como "biología" en un sentido restringido, constituye desde hace tiempo la esencia de lo que generalmente se denomina "historia natural".

El concepto de ecología en Colombia se aplica a la relación, los impactos y la atenuación con sectores representativos de la economía nacional como lo son: el sector minero, el sector energético y el sector portuario, encontrando ecosistemas intervenidos por el hombre y afectados por el deterior ambiental; pero a su vez la conciencia ambiental de las naciones ha enfilado sus esfuerzos a presentar propuestas gubernamentales que apuntan a la Sostenibilidad y a armonizar los aspectos ambientales, económicos y sociales, para el caso particular de Colombia se hace referencia a la Ley Ambiental 99 de 1993.

El objetivo del artículo es mostrar las diferentes relaciones de la ecología en Colombia en relación con los impactos y su atenuación en los sectores minero, energético y portuario; como tal se analizan los impactos positivos y negativos que se muestran alrededor de los tres sectores y las medidas atenuantes y evaluadoras de dichos impactos para presentar un resultado de las condiciones que tienen ellos.

* Magister en Desarrollo Sostenible y Medio Ambiente, Economista, Especialista en Gerencia y Mercadeo, Especialista en Administración Financiera, Especialista en Pedagogía y Docencia Universitaria. Docente Universidad la Gran Colombia en Armenia y Universidad del Quindío. Integrante Grupo de Investigación Gestión de Desarrollo del Programa de Economía "Gestión del Desarrollo"Joenpipa@ @otmail.com - Avenida Bolívar No. 7-46 Armenia, Quindío, Colombia. 
PALABRAS CLAVE

Actividades Antrópicas, Aguas de Sentina, Atmosfera, Biodiversidad, Ecología, Ecosistemas, Edáfico, Erosión, Impacto Ambiental, Minería, Procesos Morfodinámicos, Sostenibilidad, Recurso Hídrico

\title{
ABSTRACT
}

\begin{abstract}
The concept of Ecology in Colombia is applied to the relation, the impact and the mitigation of the representative sectors in national economy such as the mining sector, the energetic sector and the port's sector finding ecosystems intervened by men and affected through the environmental deterioration. But in turn the environmental conscience of nations have focused its efforts to present governmental proposals towards sustainability and to harmonize environmental, economic and social aspects, in the particular case of Colombia a reference is made to the environmental law 99, 1993.
\end{abstract}

The main purpose of this article is to show the different relations of Ecology in Colombia with the impact and the mitigation of the energetic, port and mining sector; as such, the positive and negative impacts shown around the three sectors are analyzed and the mitigating and evaluative measurements on the mentioned impacts to present a result on the conditions they've got.

\section{KEY WORDS}

Human Activities, Bilge Water, Atmosphere, Biodiversity, Ecology, Ecosystems, Soil, Erosion, Environmental Impact, Mining, Morph dynamics Processes, Sustainability, hydric Resources.

\section{INTRODUCCIÓN}

No soporta ninguna discusión, mostrar el deterioro ambiental que sufren los ecosistemas debido a la intervención del hombre sobre ellos. Cualquier acción que realiza el hombre por muy insignificante que sea, termina afectando los recursos naturales y ello se evidencia de diferentes maneras: el calentamiento global, el agotamiento de la capa de ozono, la contaminación atmosférica, la deforestación, la desertización y la salinización de los suelos, la pérdida de diversidad biológica y la contaminación de las aguas, entre otras.

Frente a esta situación que a pasos agigantados muestra el deterioro ambiental del mundo, se ha ido produciendo la generación de conciencia por parte de muchos gobiernos nacionales, además de grandes presiones de la población mundial por hacerle frente al deterioro ambiental, lo que conlleva a que en la década de los ochenta, se de el nacimiento del concepto de sostenibilidad, el cual expresa que cualquier "optimo económico debe estar asociado al equilibrio ecológico" (Brundtland 1987). Partiendo de este principio la Comisión Mundial de Medio Ambiente de la ONU, definió "el concepto de desarrollo sostenible como aquel que satisface las necesidades de la generación presente sin comprometer la capacidad de las generaciones futuras para satisfacer sus propias necesidades". (ONU 1987)
Este inicio de sensibilización frente al deterioro ambiental se profundiza en el país a partir de los años 80 del siglo XX. Colombia, uno de los países con mayor diversidad del mundo, consagra en su Constitución Política de 1991 la protección del medio ambiente y los principios del desarrollo sostenible, y mediante la ley 99 de 1993 crea el Ministerio del Medio Ambiente.

El desarrollo sostenible y armónico del mundo, requiere del equilibrio de tres aspectos fundamentales: energía, ecología y economía-social. De esta manera se estructura un enfoque en el que se armonizan, los aspectos ambientales, con los económicos y los sociales. En materia de impactos ambientales globales, principalmente emisiones de gases de efecto invernadero y sus consecuencias en el sistema climático, se evidencia la necesidad de participar de manera activa, en la elaboración, implementación y seguimiento de la estrategia nacional contra el cambio climático, en el marco de los compromisos establecidos para los países en desarrollo en la Convención de las Naciones Unidas para el Cambio Climático, la cual fue adoptada en Nueva York, en el año 1992 y entrada en vigor en el año 1994.

El proceso metodológico que se aborda en el artículo, inicia con la descripción de los efectos entre la ecología y la minería frente a la crisis ambiental, evidenciado en el incremento del uso 
de los minerales, los cuales aunque se ha convertido en renglones para la generación de empleo, producción, competitividad y desarrollos tecnológicos, han originado también pobreza y presiones sociales, económicos, además de las consabidas alteraciones ambientales. Posteriormente se aborda la información sobre los impactos originados por la actividad minera sobre los recursos agua, suelo, flora, fauna y aire; Luego se avanza sobre el análisis de la información entre ecología y sector energético, teniendo en cuenta la importancia del sistema de evaluación de impacto ambiental, los planes de desarrollo de normas y estándares ambientales, el desarrollo de políticas indicativas y los planes de descontaminación.

Seguidamente se aborda el tema ecología y sector portuario, en el cual se muestran los impactos al componente atmosférico, edáfico, hídrico, faunístico y florístico. Finalmente se muestran las conclusiones del artículo, enfatizando la importancia de los resultados para la toma de decisiones por parte de los sectores público y privado y el control y monitoreo que debe darse por parte de las autoridades ambientales y los organismos de control del país.

En la estrategia que se implementa para avanzar hacia un desarrollo energético sustentable, la introducción de energías limpias y renovables, y la promoción de la eficiencia energética, constituyen instrumentos de política energética fundamentales, por lo que urge llevar a cabo distintas acciones para el desarrollo de una política integral que, promueva la utilización de energías renovables no convencionales y haga especial énfasis en los programas de ahorro y uso eficiente de energía.

\section{LA ECOLOGIA Y LA MINERIA FRENTE A LA CRISIS AMBIENTAL}

A lo largo de la historia, Colombia ha venido incrementando el uso de los minerales y este se ha convertido en un sector que genera empleo, riqueza, pobreza, desarrollos tecnológicos, sociales, económicos y también alteraciones ambientales.

El diagnóstico es claro según lo señala el Ministerio de Minas y Energía: la minería es responsable de buena parte del deterioro de los ecosistemas, de la destrucción de gran cantidad de cuerpos de agua, de la pérdida de miles de hectáreas, de la producción de residuos tóxicos entre otros. El interrogante para países mineros como Colombia es si acaso la minería no representa un papel estratégico en el desarrollo, para atender la demanda creciente de los minerales en el mundo entero. Los minerales son unos recursos de uso diario, ampliamente utilizados para el transporte, las viviendas, la industria, los metales y piedras preciosas en la joyería, entre muchos otros minerales y usos.

Es aquí donde se ha empezado a asimilar el concepto de "desarrollo sostenible", en busca de un enfoque integral de los procesos productivos. Es importante entonces abordar con ecuanimidad el aprovechamiento y explotación sostenible de los minerales en Colombia, su potencialidad, uso, transformación, aspectos políticos y legales y los retos que en materia tecnológica, científica, social, económica, comercial, educativa y ambiental representa para nuestro país.

Al entrar a definir los impactos de la actuación del hombre sobre su entorno, necesariamente se debe de abordar la definición de categorías como la del ambiente, al respecto se puede definir que ambiente reúne elementos abióticos (suelo, agua aire, energía solar) y bióticos (organismos vivos) que conforman el espacio de la tierra donde tiene lugar la vida.

Una de las clasificaciones más utilizadas para abordar el estudio de lo ambiental es la referida por (Hernández 2009) el cual manifiesta: "que el ambiente puede ser abordado desde las siguientes disciplinas y dimensiones del conocimiento:

- Física: disciplinas de geografía, geología, física, química, geometría, matemática, entre otras.

- Biológica: Disciplinas de zoología, botánica, fisiología, etiología.

- Antrópicas: Disciplinas de economía, historia, culturales, sociológicas y ecológicas".

En la primera mitad del siglo XX siglo, la ecología como ciencia biológica, se ocupó de establecer los fundamentos científicos para entender la unidad de la vida. Producto de la creciente sensibilización de la humanidad con relación a los crecientes problemas del hombre y su relación 
con el medio ambiente, a partir de los años 30 del siglo pasado se da una dimensión diferentes a categorías que tenían una aplicación mas restrictivas a determinadas esferas científicas, una de ellas es la de la ecología, la cual presentaba como límites las llamadas ciencias naturales, abordando otras relaciones, que más tarde se llamarían por ejemplo la "ecología humana", trascendiéndola al estudio de otras actividades por ejemplo de la economía, dando surgimiento una nueva dimensión y significado, ubicando la ecología como la disciplina científica que se que ocupa del estudio de la economía ambiental, es decir, como cuando señala Hernández, la variable costo-beneficio forma parte directa de los análisis y estudios ambientales.

Actualmente la minería es una de las actividades más contaminantes y puede tener los siguientes impactos en el ambiente: en actividades de exploración, explotación y transporte de minerales como carbón, auríferas, contaminación por actividades de carbón, petróleo, por emisiones sólidas, líquidas o gaseosas, deterioro por uso inadecuado del suelo y destrucción paisajística.

\subsection{La minería y el deterioro ambiental}

En términos generales, la minería constituye una actividad económica que requiere de poca mano de obra, siendo intensiva en el uso de capital. En relación a la agricultura, industrias o servicios, la actividad minera tampoco es una gran generadora de ingresos directos. Como sucede con otras formas de producción extractiva, la localización y las características de las empresas mineras se encuentran influidas por factores espaciales y ambientales que interactúan entre sí.

Los beneficios económicos que pueden derivarse de una reserva minera están relacionados con su accesibilidad (incluyendo su proximidad a los grandes mercados industriales) y con su calidad (referida al tamaño, forma, profundidad y ley del mineral).

Según el Ministerio de Minas y Energía, en la ubicación de la mina existen dos maneras de desarrollar la actividad:

- Mina a cielo abierto: El proceso extractivo de estas minas se realizan sobre la superficie del terreno. En este tipo de extracción se va excavando con grandes maquinarias para obtener los metales; las minas a cielo abierto son las más contaminantes porque durante el proceso de extracción la roca vuela provocando nubes de polvo, que son arrastradas por los vientos cordilleranos y transformados en lluvia ácida.

- Mina subterránea o de socavón: Se utiliza una maquinaria más pequeña ya que de trabaja en túneles y galerías; la minería subterránea puede contaminar las aguas freáticas de diversas maneras, las aguas de mina son una fuente importante de contaminación, al igual que las soluciones utilizadas en la lixiviación; la contaminación con polvo puede provocar diferentes enfermedades, de las cuales la más peligrosa es la silicosis causada por la inhalación de partículas de sílice y el polvo es producido por la destrucción de las piedras y montañas.

Las actividades mineras son responsables de un alto porcentaje de las modificaciones del paisaje natural. Entre los impactos que en la minería, mas grandemente afectan el medio ambiente están: los cambios en la calidad físico-química del agua, la remoción y pérdida del suelo, la generación de escombros, la remoción y pérdida de cobertura vegetal (y por consiguiente el desplazamiento de comunidades bióticas), los cambios en el uso del suelo y la modificación de la armonía y dinámica del paisaje.

El decreto 2820 de 2010 reglamenta el titulo VIII de la Ley 99 d e1993 "De creación del Sistema Nacional Ambiental y el Ministerio del Medio Ambiente y en cuanto a la explotación minera determina que el Ministerio de Ambiente, Vivienda y Desarrollo Territorial, otorgará o negará de manera privativa la licencia ambiental para los siguientes proyectos, obras o actividades:

- Carbón: Cuando la explotación proyectada sea mayor o igual a 800.000 ton/año.

- Materiales de construcción y arcillas o minerales industriales no metálicos: Cuando la producción proyectada sea mayor o igual a 600.000 ton/año para las arcillas ó mayor o igual a $250.000 \mathrm{~m} 3 /$ año para otros materiales de construcción o para minerales industriales no metálicos. 
- Minerales metálicos y piedras preciosas y semipreciosas: Cuando la remoción total de material útil y estéril proyectada sea mayor o igual a 2.000 .000 de ton/año.

- Otros minerales y materiales: Cuando la explotación de mineral proyectada sea mayor o igual a 1.000 .000 ton/año.

En este sentido, en el año 2009 los Ministerios de Ambiente, Vivienda y Desarrollo Territorial y Minas y Energía con el propósito de actuar en la mitigación de los impactos ambiéntales, diseñaron una metodología que facilita el estudio necesario para su evaluación, se realiza en primer lugar una correlación de las actividades realizadas para construcción, montaje y explotación del proyecto minero con cada uno de los efectos potenciales. Luego se procede a la valoración de los impactos, de acuerdo a criterios cualitativos, para establecer una magnitud; finalmente se hace referencia a las medidas de manejo ambiental de acuerdo con la relación específica de las actividades generadoras del impacto o los impactos.

Con en el objeto de facilitar la identificación y control de los impactos ambientales, la guía minera ambiental los clasifica por su origen.

\section{Impactos sobre el suelo}

Cuando las exploraciones mineras tienen que realizar movimientos de macizo rocoso a poca profundidad y producto de un soporte inadecuado, generan hundimientos del terreno que alteran abruptamente la topografía, produciendo deslizamientos o movimientos masales del terreno. De otra parte cuando se trata de minería profunda los hundimientos son lentos y de contorno suave, y son llamados por los técnicos como subsidencia.

Otro impacto generado en la etapas de la exploración y explotación hace referencia a que la perdida de la cobertura vegetal y la capa superficial del suelo en terrenos con pendiente pronunciada, el cual una vez expuesto a la lluvia y al sol, generan desprendimientos de material a corto, mediano y largo plazo, produciendo los procesos de degradación del suelo.

\section{Impactos sobre la calidad del agua}

Estos impactos están relacionados con la alteración de la calidad físico química de las aguas, incremento de la turbidez por aportes de sólidos suspendidos o disueltos, modificación del drenaje natural, colmatación de cuerpos de agua, variación de los niveles freáticos, afectación de la dinámica de cuerpos de agua subterráneos y superficiales, sedimentación en cuerpos de agua, disminución del caudal y vertimientos de aguas residuales mineras, domésticas, industriales y disminución del caudal.

\section{Impactos en el componente flora}

El impacto esta determinado por la remoción y pérdida de cobertura vegetal. La cobertura vegetal se puede afectar por diversas maneras durante las actividades de construcción, montaje y adecuación de áreas para la explotación. Con la pérdida de especies vegetales se disminuye la biomasa vegetal, se altera el paisaje, se modifica el hábitat para la fauna, se aceleran o se inducen procesos erosivos, y se puede alterar la calidad y cantidad de aguas.

La minería subterránea puede generar reducción del ecosistema boscoso, en cuanto se requiera madera para el entibado de la mina. Este se hace más evidente en las etapas de construcción y montaje del proyecto minero, perforación y voladura, extracción del mineral y la disposición de escombros.

\section{Impactos en el componente fauna}

Este es un impacto que se genera en todas las etapas de ejecución del proyecto minero, están determinadas principalmente por la alteración y disminución de hábitats por perdidas de cobertura vegetal, dispersión o fuga de algunos individuos debido al incremento del ruido por las actividades de explotación, aumento de accidentalidad debido al incremento del tráfico vehicular y por el incremento de la cacería sobre especies de valor comercial por parte del personal vinculado al proyecto.

\section{Impactos en el componente aire}

Este se determina principalmente por el aumento de material particulado y gases, se origina principalmente en la construcción de vías, cargue y transporte del mineral. También se produce por la operación de maquinaria y equipos de explotación, por la manipulación del mineral o por la acción del viento sobre las pilas del mineral en los patios de acopio; así mismo, la descomposición de la roca $y$, en algunos casos, las voladuras liberan a la atmósfera material particulado y gases. 
Hruscka (2006), señala algunos costos generados en especial por la pequeña y mediana minería:

- Los aspectos geológicos relacionados con la explotación de los yacimientos, los cuales conducen finalmente a su extinción.

- Las consecuencias para los recursos naturales en su totalidad.

- Los aspectos sociales que determinan opciones de mejorar nivel de vida en detrimento de calidad de vida.

Otros aspectos que destaca el autor se pueden resumir en estos cuatro campos al realizar la evaluación, ya sea esta cualitativa o cuantitativa, lo más importante es tener presente la situación local, es decir:

- La situación geológica relacionada con la explotación de los yacimientos.

- La situación medio-ambiental que origina desbalances en el ecosistema.

- La situación social en las inmediaciones de la pequeña minería.

- Las condiciones económicas a nivel regional y nacional.

Como estas condiciones locales básicas varían en cada caso, también variarán los resultados de una evaluación de efectos idénticos de la pequeña minería serán diferentes. Una explotación a cielo abierto ubicada en el desierto del norte chileno ha de ser valorada de otra manera que una explotación situada en un sistema ecológico digno de ser protegido. Con esto ya queda evidente que, al considerar los costos y beneficios de la pequeña minería en los países en desarrollo, no se puede generalizar ni siquiera para realizar la valoración.

Así mismo señala algunos beneficios, los cuales se resumen de la siguiente manera.

- Movilización de recursos nacionales.

- Recaudo de impuestos.

- Efecto activador para la balanza de pagos.

- Ofrece reservas de personal para la minería industrial.

- Contribución al desarrollo económico regional por:

- Circulación monetaria (producto social)

- Inversiones.
- Demanda de productos y prestaciones de servicio.

- Movilidad.

- Consecuencias estructurales (alternativa frente a la agricultura).

- Se evita el éxodo rural.

- Desarrollo de la infraestructura (construcción de carreteras, escuelas, abastecimiento de energía) por la pequeña minería y población aledaña.

- Ventajas financieras comparativas (producción con alto coeficiente de mano de obra en países con una gran oferta de mano de obra).

- La oferta del producto es relativamente estable aún en caso de fluctuaciones del mercado.

- Aporte a la diversificación de productos y de la exportación.

Como se puede observar en esta comparación de costos y beneficios, los primeros afectan directamente los recursos naturales y la población, los segundos benefician a los inversionistas y en términos macroeconómicos a las estadísticas del país.

\subsection{Manejo de impactos ambientales}

Los altos impactos ambientales señalados anteriormente obliga a que las autoridades ambientales y los ejecutores de los proyectos mineros, para responder de manera adecuada a las obligaciones y responsabilidades de carácter ambiental que se derivan de su ejecución tengan claridad y conocimiento de la planificación y el manejo ambiental de un proyecto minero, de su naturaleza y las implicaciones del mismo; a esta obligación socioambiental la mejor respuesta es la formulación y ejecución de una manera lógica, del Sistema de Gestión Ambiental (SGA) del proyecto minero.

Como se mencionó anteriormente los ministerios de Ambiente, Vivienda y Desarrollo Territorial y Minas y Energía, elaboraron una guía para la gestión ambiental, la cual obliga a los mineros asumir compromisos en entorno a la prevención de impactos ambientales. Esta guía ambiental, tiene cinco componentes que son: políticas y normatividad, análisis ambiental, medidas de manejo ambiental, monitoreo y seguimiento y evaluación y mejoramiento. 
El establecimiento y ejecución de una política ambiental es una manera de hacer explícito el compromiso del sector minero, con el cumplimiento de la normatividad y con la eficiencia de la gestión ambiental, en concordancia con la viabilidad técnica y económica de la actividad. La política ambiental además de ser dinámica requiere que sea divulgada, conocida y aplicada por todos los niveles del proyecto minero como única forma de atenuar todos los impactos ambientales que esta actividad le genera al país.

(Weber J. L.2005), presenta una propuesta para valorar los recursos naturales y lograr que se recuperen los daños ocasionados a ellos, es corregir los valores monetarios de la contabilidad nacional, particularmente la Renta Nacional y el Producto Interno Bruto, con el fin de incorporar de forma real no solo los aspectos macroeconómicos sino además los microeconómicos y entre estos últimos plantea la posibilidad de desarrollar las cuentas del patrimonio económico como son las cuentas satélites y físicas del medio ambiente para lograr valorar realmente la perdida o ganancia en el aprovechamiento de los recursos naturales. Esta seria la mejor manera de lograr acercarnos a la contabilidad ambiental.

\section{ECOLOGIA Y SECTOR ENERGETICO FRENTE A LA CRISIS AMBIENTAL}

La energía constituye un insumo vital para el desarrollo del país, sin embargo, su producción, transporte y consumo habitualmente presenta impactos ambientales de diferentes niveles de significancia, y cuyos efectos pueden ser de carácter local o global. El sector energético reconoce la existencia de estos impactos, y por ello la necesidad de incorporar la sustentabilidad ambiental como uno de los elementos claves de la estrategia de desarrollo integral del sector.

En el ámbito local, algunos elementos que forman parte de la dimensión ambiental en el sector energético como lo señala el Ministerio de Minas y Energía son los siguientes:

- Sistema de Evaluación de Impacto Ambiental.

- Planes de desarrollo de normas y estándares ambientales.
- Desarrollo de políticas indicativas.

- Planes de descontaminación.

El crecimiento de la economía nacional, es un factor que inevitablemente lleva a un aumento del consumo energético presionando por un aumento de la oferta energética disponible. Ello se traduce en la ocurrencia de mayores conflictos ambientales derivados de la generación, distribución y uso de la energía.

El desafío de incorporar variables de sustentabilidad en el desarrollo del sistema energético, requiere conciliar el abastecimiento de la creciente demanda de energía, con una protección efectiva del medio ambiente Esto implica avanzar en la equidad intergeneracional, que permita asegurar el derecho de las generaciones venideras por vivir en un medio ambiente sano y libre de contaminación, y al mismo tiempo, no frenar el justo anhelo de alcanzar mejores niveles en la calidad de vida y de un mayor progreso para las actuales generaciones de nuestro país.

Hacer un uso eficiente de la energía surge en este escenario, como un requisito ineludible de todos los actores del mercado energético: productores, consumidores, reguladores, y es una solución concreta que contribuye a una mayor equidad intergeneracional, a mejorar la competitividad de la economía, disminución de impactos ambientales derivados de una menor producción y consumo de energía, y a reducir a lo estrictamente necesario las expansiones que naturalmente requiera el sistema energético nacional.

La producción, transporte y consumo de energía genera importantes impactos ambientales. En el ámbito local, un primer elemento lo constituye el proceso de evaluación ambiental al cual los megaproyectos energéticos deben someterse, con el objeto de minimizar los impactos ambientales de su desarrollo (ONU 2008).

Con relación a los efectos globales, es necesario reconocer la importancia del sector para minimizar los efectos de las emisiones de gases de efecto invernadero y sus consecuencias en el sistema climático, promoviendo una activa participación del sector energético en la estrategia global diseñada por las Naciones Unidas para combatir este fenómeno. 
La evolución en los últimos años de la intensidad eléctrica, indica que se requiere cada vez más electricidad por unidad de PIB Ministerio de Minas y Energía (2009). Sin embargo, es necesario señalar las reservas con que debe ser utilizado un indicador global como éste, dado que no es posible obtener conclusiones sobre la evolución de la eficiencia energética.

En efecto, cambios en la intensidad energética o eléctrica se pueden explicar por muchos factores tales como: cambios en la estructura productiva del país, modificaciones en la matriz energética, factores climáticos y cambios en la eficiencia energética, entre otros.

\subsection{Atenuación de impactos}

Para poder determinar los impactos que el sector energético ejerce sobre el medio ambiente es importante conocer el ciclo de vida de este sector.

SISTEMA ELECTRICO DE POTENCIA Energía Eléctrica

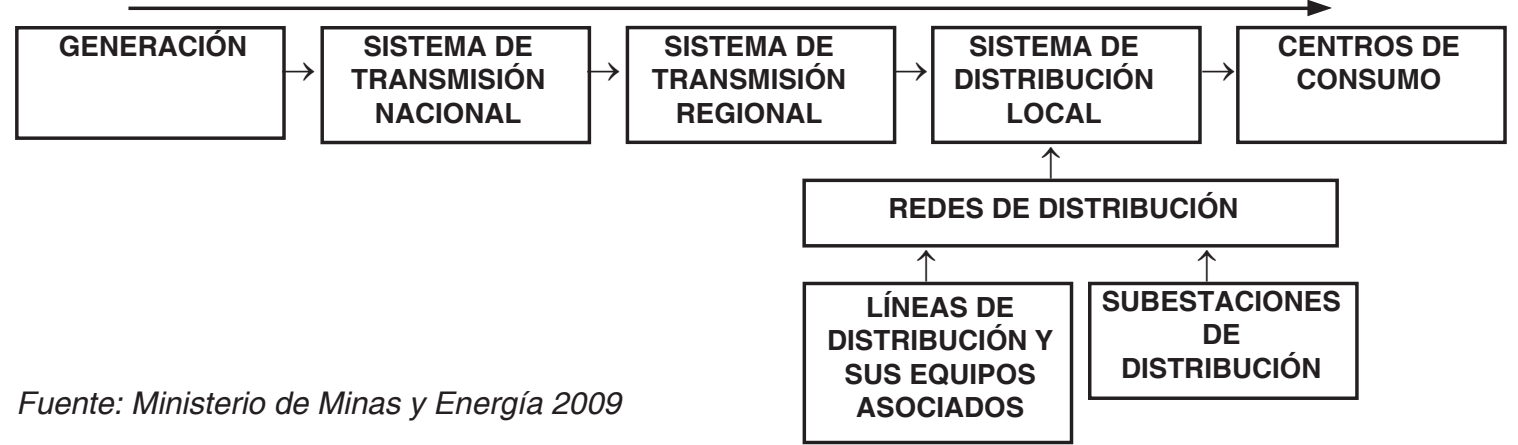

En la gráfica se puede observar que son varios los procesos que en el sistema eléctrico existen; generación, transmisión y distribución de la energía eléctrica y su función primordial es la de trasladar esta energía desde las centrales de generación, para su aplicación en los centros de consumo, en cada uno de estos procesos son varios y diferentes efectos que se generaran en el medio ambiente.

Cada una de las actividades que realiza en el sector eléctrico produce diferentes impactos, los más significativos que potencialmente ocurren en la construcción, operación, mantenimiento y desmantelamiento de una línea de distribución de energía son:

\section{En el medio social}

- Generación de Expectativas.

- Generación de Empleo (Mano de Obra No Calificada del Área de Influencia del Proyecto).

- Afectación del Patrimonio Cultural (Arquitectónico, Arqueológico y Natural).

- Afectación al Uso del Suelo.

- Afectación de Infraestructura Vial y de Servicios Públicos.

- Incremento del Riesgo de Accidentalidad.

- Afectación a la Comunidad.

- Obstrucción del Tráfico Vehicular. 


\section{En el medio natural}

- Erosión.

- Desestabilización de Taludes.

- Generación de Residuos Sólidos y Material Inerte.

- Afectación a Cuerpos de Agua.

- Afectación de Nacimientos o Sitios de Captación.

- Afectación de la Cobertura Vegetal.

- Fragmentación de Ecosistemas y Efecto de Borde.

- Afectación de Bosques Primarios.

- Alteración y/o Disminución de Fauna Silvestre.

- Afectación del Paisaje.

- Generación de Residuos Aceitosos.

Todas estas causas afectan en gran medida parcial y definitivamente los ecosistemas pero con la realización de medidas sobre la ecología, que se define como la rama de las ciencias biológicas que se ocupa de las interacciones entre los organismos y su ambiente (sustancias químicas y factores físicos), podemos mitigar algunos de estos efectos.

El estudio integrado de la ecología nos proporciona una serie de elementos que nos ayudan a restaurar ecosistemas afectados, ya que la ecología se relaciona con diferentes disciplinas como la geología, climatología, física, matemáticas, geografía, entre otras y por tal razón el estudio de la ecología es fundamental para poder formular soluciones en ecosistemas alterados por el sistema de generación eléctrica y de otros sistemas productivos (Holdridge 2000).

Son variadas las opciones que el Ministerio de Minas y Energía propone y que se pueden producir para disminuir los impactos que genera el sector eléctrico en el país, algunos de ellos pueden ser:

- Identificar, evaluar y proponer medidas e instrumentos de eficiencia energética, para los distintos sectores de consumo del país.

- Elaborar una propuesta de regulación para Implementar un programa de certificación y etiquetado de eficiencia energética.

- Desarrollar un programa integral que permita caracterizar el comportamiento del consumidor residencial, industrial y comercial y su disposición a incorporar aspectos de eficiencia energética en sus decisiones y hábitos.

- Intensificar los procesos de investigación para el uso de fuentes no convencionales de energía, son las que, por falta de avance tecnológico o por sus cuantiosos gastos de extracción y aprovechamiento, aún son limitadas; algunas de ellas pueden ser: energía mareomotriz, energía eólica, energía de biomasa, energía solar, energía nuclear, energía geotérmica, gas natural, biogás, entre otras.

- Proporcionar fácil acceso a servicios energéticos sostenibles; Para ello, es necesario avanzar en al reducción de la pobreza y alcanzar objetivos sostenibles de desarrollo en los ámbitos local, regional y nacional. Ello debe incluir la promoción de tecnologías de energía limpia para disminuir los cambios climáticos y aumentar el acceso a la financiación de inversiones en energía sostenible, incluyendo el Mecanismo de Desarrollo Limpio, la gestión sostenible de la tierra para combatir la desertificación y la degradación de la tierra y la conservación y uso sostenible de la biodiversidad.

- Diseñar y aplicar una política nacional y sectorial y planificación del control de emisiones de substancias que dañan el ozono (ODS) y de substancias orgánicas que causan polución persistentemente (POP). Se ayuda a las empresas a mantener su competitividad económica dándoles las mejores tecnologías alternativas que existen y oportunidades para el desarrollo de sus capacidades.

Todas las acciones que se realizan deben procurar en restablecer y restaurar los ecosistemas, por eso el estudio de la ecología es tan importante para poder identificar claramente las medidas a realizar en cualquier contexto, en este caso el sector eléctrico.

La ecología es una herramienta efectiva que nos puede proporcionar orientaciones en las diferentes medidas ambientales que se efectúen en ecosistemas alterados y logra facilitar y optimizar el proceso de gestión ambiental a través de las diferentes fases del ciclo de vida de un proyecto de distribución eléctrica (Ministerio del Medio Ambiente, 1997). 
La utilización de la ecología se puede alcanzar diferentes objetivos específicos para mejorar, mitigar y conservar ecosistemas alterados y para nuestro caso especifico mejorar los sistemas de gestión ambiental en el sector eléctrico, se tienen los siguientes:

- Unificar criterios ecológicos y fortalecer la gestión ambiental.

- Realizar una planificación ambiental efectiva con criterios ecológicos.

- Unificar criterios de evaluación ambiental y mejorar los ecosistemas.

- Planificar de forma analítica y coherente una serie de medidas típicas de manejo, control y seguimiento ambiental que parte del análisis de los efectos de un proyecto.

- Relacionar las acciones ecológicas que se planifiquen para la gestión ambiental con la legislación y normatividad ambiental vigente para cumplimiento y aplicabilidad.

Estos objetivos están directamente relacionados con el conocimiento de la ecología, y vislumbran una serie de acciones que están íntimamente ligados a sus principios y sus interacciones (Ministerio del Medio Ambiente, 1997).

\section{ECOLOGIA Y SECTOR PORTUARIO FRENTE A CRISIS AMBIENTAL}

El concepto de actividad portuaria es definido en el Art. 5 de la Ley 01/91 así: "Se consideran actividades portuarias la construcción, operación y administración de puertos, terminales portuarios; los rellenos, dragados y obras de ingeniería oceánica; $y$, en general, todas aquellas que se efectúen en los puertos y terminales portuarios, en los embarcaderos, en las construcciones que existan sobre las playas y zonas de bajamar..."

El concepto de puerto implica la consideración de dos escenarios: el acuático y el terrestre. El medio acuático se puede referir al ecosistema marino o al fluvial, en él se construyen obras (rompeolas, muelles) y se establecen las estructuras (p.ej: bandas transportadoras) requeridas para el acceso y circulación de las embarcaciones (buques, barcazas, remolcadores) y para el manejo y transferencia del mineral. En tierra se disponen las instalaciones de apoyo técnico, logístico y administrativo del puerto, las áreas de recepción y acopio temporal del mineral, así como todo lo relacionado con los servicios y suministros de los que se precisa para garantizar la operación normal de un puerto carbonífero.

Con el modelo de concesiones portuarias, los puertos del país pasaron de ser un monopolio público ejemplo de sobrecostos e ineficiencia, a un modelo de gestión en América Latina. Con su privatización, el valor real de las tarifas portuarias disminuyó, en promedio, el $65 \%$, quedando a niveles competitivos internacionales; los fletes se redujeron, los tiempos promedio de estadía de las mercancías se redujeron de 10 días a 12 horas gracias a tecnologías de comunicaciones que conectan en tiempo real a la aduana, los depósitos, las líneas marítimas, los transportadores terrestres y demás actores de comercio exterior con el puerto y a una infraestructura moderna y dotada de equipos de última tecnología que permiten la manipulación más rápida, segura y eficiente de las cargas. (CORPES de Occidente 1999).

\subsection{Impactos y propuestas ambientales para el sector portuario}

La propia mejora del entorno en que se localizan las instalaciones de los puertos y la búsqueda del aumento en la eficacia de los procesos que se desarrollan en la instalaciones es uno de los difusores de impactos ambientales; no obstante los mas significativos son los que están relacionados con la actividad portuaria como el desarrollo de proyectos de puertos, el cual implica en sus primeras etapas la definición de la infraestructura requerida para recibir, manejar y exportar los materiales que se van a manejar a través del puerto y las típicas de un proyecto de ingeniería, que incluyen básicamente: factibilidad, diseño, construcción, operación y cierre.

Es importante señalar la importancia que hoy cobra para este tema de disminución de impactos ambientales el Convenio Internacional para la Prevención de la Contaminación generada por Buques MARPOL, el cual determina "que los Estados Parte en el mismo se comprometen, entre otras cosas, a que en los puertos u otros lugares de la costa bajo jurisdicción donde realicen operaciones los buques, deben existir facilidades para la recepción de los desechos generados a bordo, implica que las "instalaciones deben ser las adecuadas para 
el tráfico marítimo que soportan los puertos y el volumen de residuos que esperan recibir, que dependerán de las rutas y el tipo de buques facilitando así el tráfico marítimo internacional (Marpol. net 1973).

Siendo así los siguientes aspectos determinados en los componentes del medio ambiente determinan la identificación y corrección de impactos:

\section{Componente atmosférico}

- La construcción del proyecto, las labores de desmonte, limpieza, descapote, remoción de la capa de suelo y adecuación de vías, contribuirán directamente al aumento de material particulado.

- La etapa de operación, las actividades de manejo: descargue de tractomulas, tren o barcazas, conformación de pilas, acopio transitorio, recuperación del mineral desde el sitio de acopio hasta el cargue de barcazas y de ahí a los buques, la circulación de vehículos en las áreas de operación; incrementarán los niveles de material particulado.

- El aumento de las concentraciones de gases por la utilización de maquinaria y equipo pesado en las actividades de construcción del proyecto aumentarán las concentraciones de gases como: SOx, NOx, COx, HC.

- El nivel de ruido por la utilización de maquinaria y equipo pesado en las actividades de construcción del proyecto, así como durante la operación del puerto pueden ocasionar el incremento de los niveles comunes de ruido, principalmente en el área interna del puerto.

En este contexto, la siguiente es la propuesta para atenuar estos impactos:

1. Aplicar de manera técnica los procedimientos o métodos de control de las emisiones de partículas, los más usados son:

- Sistema de aspersión en tolvas de recibo, en pilas y patios de acopio y en puntos de descarga de la banda transportadora.

- Cubrimiento de la banda transportadora.

- Control de velocidad de vehículos livianos.

- Humectación continúa de vías de tránsito de equipo liviano y pesado.
- Establecimiento de barreras vivas en patios de acopio.

- Establecimiento de cobertura vegetal en áreas descubiertas.

2. Para el control de niveles de ruido se plantea como objetivo adoptar medidas o acciones con el fin de prevenir, controlar y mitigar la contaminación debida a la emisión de ruido, como las relacionadas enseguida:

- Identificación de los puntos y medición de niveles de ruido.

- Instalación de aislamientos sonoros en los puntos críticos.

- Amortiguación de estructuras en tolvas de recibo y bandas transportadoras.

- Eliminación de resonancias en tolvas y bandas transportadoras.

\section{Componente edáfico}

- Disminución del volumen de biomasa.

- Alteración de la calidad del paisaje.

- Modificación del hábitat para la fauna silvestre de la región.

- La inducción o aceleración de procesos erosivos.

- La alteración de la calidad y la cantidad de agua.

- Desestabilización de laderas.

- Cambios en el uso de suelo.

- Contaminación por derrames de combustibles, grasas y aceites.

\section{Esta problemática puede ser corregida así:}

1. Aumento de la cobertura vegetal: el manejo de este impacto se puede lograr de dos formas: (i) minimizando las áreas en donde se removerá vegetación, de tal manera que la pérdida de la cubierta vegetal sea la menor posible y (ii) efectuando una restitución de la capa vegetal. En la etapa inicial se puede realizar la siembra de especies gramíneas y posteriormente, a mediano y largo plazo, la implantación de especies arbóreas y arbustivas.

2. Estabilización de áreas: el estudio geológico y geotécnico suministra la información pertinente para evitar la intervención de áreas frágiles, cuya alteración pueda causar desestabilización 
de los terrenos. De otra parte, el conocimiento de las estructuras geológicas también será necesario para diseñar las obras de control y estabilización de las áreas intervenidas, para ello se requiere:

- Cambios en el uso del suelo: es una actividad sin equa non para la realización de este tipo de proyectos. El manejo de este impacto requiere el planteamiento de medidas compensatorias, por efecto de las pérdidas en actividades agrícolas, turísticas o en general, de las actividades económicas que se realicen con anterioridad al emplazamiento del proyecto portuario.

- Desarrollo de procesos erosivos y de sedimentación en la costa: será función de las características del medio marino (oleaje, corrientes, vientos, dinámica natural etc) y de las especificaciones de las obras a construir (rompeolas, dársenas, tajamares etc). El manejo del impacto requiere la adopción de diseños funcionales que minimicen la interferencia de las corrientes marinas y, posteriormente, durante la operación, será necesaria la programación de actividades de mantenimiento (dragados) para remover excesos de sedimentos en proximidades de las obras.

- Mejoramiento estético de las playas: es inevitable que cantidades menores de elementos se dispersen en las inmediaciones de un puerto carbonífero y sedimenten en las playas próximas a las áreas de manejo carbón. Desde el punto de vista del control del impacto, la utilización de barreras (vivas o artificiales) podrán disminuir la cantidad de carbón sedimentable. Como acción remedial se recomienda efectuar limpiezas periódicas (manuales o con maquinaria) para retirar el carbón de las superficies más afectadas.

- Manejo de la contaminación por derrames de combustibles, grasas y aceites: el control preventivo de este impacto requiere esencialmente la adopción de prácticas adecuadas de manejo de combustibles y lubricantes en las áreas de mantenimiento y de suministro de los mismos. Las acciones remediales incluyen la limpieza y remoción de áreas contaminadas mediante la utilización de arena o tierra como medio absorbente y su posterior disposición en los sitios diseñados con este fin.

\section{Componente hídrico}

- Deterioro de la calidad de agua.

- Aportes de sedimentos durante la construcción del puerto.

- Vertimientos líquidos y desechos sólidos.

- Transporte y deposito de elementos del puerto.

- Aguas de sentina y otros contaminantes.

- Afectación de la dinámica fluvial y de los patrones de drenaje natural.

Esta serie de problemas pueden ser manejados así:

1. Manejo del Recurso Hídrico, mediante:

- Control y Manejo de aguas de escorrentía no contaminadas.

- Control de las aguas de escorrentía superficial del área del puerto.

- Evitar el aporte de sedimentos a los cuerpos de agua.

- Evitar la acumulación de aguas lluvias y la consecuente proliferación de insectos vectores.

- Construcción de canales perimetrales recolectores.

- Construcción de lagunas de sedimentación.

2. Manejo de Aguas Residuales Domésticas, teniendo en cuenta que:

- En la mayoría de los casos es posible lograr un tratamiento adecuado con un sistema conformado por tanque séptico, filtro anaeróbico y el correspondiente campo de infiltración o pozo de absorción.

- En forma alternativa, se pueden tratar las aguas residuales en plantas compactas (lodos activados) o de una manera más sencilla en lagunas de oxidación.

3. Manejo de Aguas Residuales Industriales, mediante:

- Construcción de canales recolectores de aguas de escorrentía superficial.

- Construcción de sedimentador de lodos. 
- Construcción de piscina de lodos.

- Construcción de trampa de grasas.

- Construcción de laguna de sedimentación.

4. Manejo de Aguas de Sentina:

Uno de los elementos sensibles del control ambiental tiene que ver con el manejo de sustancias a bordo de los buques o de las facilidades portuarias. Al respecto, vale la pena mencionar que en la Legislación Nacional se establece la forma como deben manejarse estos productos y que Colombia ha firmado convenios internacionales en los que se compromete a cumplir con las normas de seguridad y protección del ambiente, otorgando derechos a la nación para exigir que embarcaciones que llegan a sus puertos cumplan con estas mismas normas, que se encuentran reglamentadas a través de la Dirección General Marítima dentro del convenio.

\section{Componente de aguas de sentina}

- Afectación de los ecosistemas de fauna y flora presentes en la zona.

- Menoscabo de la capacidad de especies faunísticas y florísticas de asimilar los cambios producidos por el puerto.

- Desaparición de especies que se encuentran amenazadas o en peligro de extinción.

- Alteración del paisaje, migración de fauna, y aceleración de procesos morfodinámicos.

Esta serie de problemas pueden ser manejados así:

- Evitar la pérdida del suelo fértil removido, con un manejo adecuado durante el proceso de construcción del puerto.

- Establecer una cobertura vegetal oportuna sobre sitios críticos en donde se puedan presentar procesos erosivos.

- Compensar la pérdida de vegetación de las áreas de construcción, plantando en otros lugares las especies afectadas y reforestando áreas degradadas con anterioridad.

- Repoblar y enriquecer la vegetación.

- Establecer cercas vivas y barreras cortavientos.

- Construir sitios de paso para el desplazamiento de la fauna o en su defecto comple- mentar las áreas cortadas con abrevaderos, refugios, comederos, etc.

- Reducir el impacto visual.

- Las medidas de manejo de la vegetación sugeridas son:

- Minimizar la tala de árboles en el área del proyecto.

- Limitar el desmonte a las áreas estrictamente necesarias para la operación del puerto.

- Realizar programas de reforestación con especies nativas.

- Realizar programas de educación ambiental.

\section{Componente fauna y flora}

- Afectación de los ecosistemas de fauna y flora presentes en la zona.

- Menoscabo de la capacidad de especies faunísticas y florísticas de asimilar los cambios producidos por el puerto.

- Desaparición de especies que se encuentran amenazadas o en peligro de extinción.

- Alteración del paisaje, migración de fauna, y aceleración de procesos morfodinámicos.

Esta serie de problemas pueden ser manejados así:

- Evitar la pérdida del suelo fértil removido, con un manejo adecuado durante el proceso de construcción del puerto.

- Establecer una cobertura vegetal oportuna sobre sitios críticos en donde se puedan presentar procesos erosivos.

- Compensar la pérdida de vegetación de las áreas de construcción, plantando en otros lugares las especies afectadas y reforestando áreas degradadas con anterioridad.

- Repoblar y enriquecer la vegetación.

- Establecer cercas vivas y barreras cortavientos.

- Construir sitios de paso para el desplazamiento de la fauna o en su defecto complementar las áreas cortadas con abrevaderos, refugios, comederos, etc.

- Reducir el impacto visual, teniendo en cuenta las condiciones de la guía.

- Las medidas de manejo de la vegetación sugeridas son:

- Minimizar la tala de árboles en el área del proyecto. 
- Limitar el desmonte a las áreas estrictamente necesarias para la operación del puerto.

- Realizar programas de reforestación con especies nativas.

- Realizar programas de sensibilización, formación y educación ambiental.

\section{CONCLUSIONES}

Como parte de las políticas ambiéntales nacionales, actualmente es requisito la realización de estudios de impacto previos a la aprobación de obras y proyectos de inversión. Sin embargo, esto no ha impedido la aprobación de proyectos, caso en el sector minero el proyecto mina "La Colosa" en Cajamarca Tolima, que afectan recursos naturales estratégicos como la provisión de agua, degradación del suelo y afectación de la fauna y flora en importantes ecosistemas alto andinos colombianos.

En diferentes municipios del país se presentan resistencia de las comunidades pobladoras de esas regiones a la realización de proyectos mineros por el gran impacto negativo en los aspectos ambientales y culturales-religiosos que estos generan en las comunidades y su entorno, por ejemplos en los municipios de Carmen del Darién, por la objeción de la mayoría de las comunidades indígenas expresan que no están de acuerdo ni con la exploración ni la explotación del Cerro, en una mina a cielo abierto para la extracción de molibdeno, oro y cobre; la misma postura la han asumido las organizaciones de los pueblos indígenas, mestizos que habitan en el Catatumbo respecto a la extracción carbonífera.

Hoy, es imposible no reconocer las consecuencias ambientales de la actividad minera, en la tierra todo está interconectado, cada ser viviente, no importa su especie, está relacionado con otros muchos y existe un entorno común en el cual las afectaciones son irreversibles para toda la población humana como animal y vegetal.

Los sectores portuario y energético aunque desarrollan una importante gestión que permite a la nación integrar procesos para avanzar hacia la competitividad internacional, aun no ha comprendido la importancia estratégica de la base natural en la cual se soportan los proyectos de desarrollo; ello ha conducido a que se limiten a cumplir con leyes y normas puntuales, dejando de lado que el sistema natural es integral y como tal su funcionamiento es de carácter holístico y como tal su manejo es completo como proceso y no aislado como unidad.

\section{RECOMENDACIONES}

Se debe realizar la gestión minera, energética y portuaria, entendiendo que la gestión las organizaciones comprende los procesos de planificación, administración y la financiación de manera integral y no aislados cada uno de estos componentes; para ello se requiere y teniendo en cuenta la dimensión ambiental que los cobija, se requiere utilizar sistemas de manejo, procedimientos, procesos y/o tecnologías que contribuyan a la eficiencia minera, energética y portuaria, valorando de manera efectiva el uso de las recursos naturales, con el fin de minimizar los efectos ambientales y coadyuvar a fortalecer el sector empresarial del país y mejorar la calidad de vida de la sociedad.

Se deben reconocer los fundamentos, parámetros de evaluación y análisis que deben ser llevados a cabo para una gestión minera, portuaria y energética, reconociendo los esquemas de planificación ambiental, para que sea entendida como un proceso de articulación de los sectores que identifica oportunidades para mejorar la eficiencia de ellos y con el fin de disminuir los efectos e impactos ambientales en cada una de las fases de los proyectos o procesos de él; para ello se requiere utilizar tecnologías de reducción, mitigación, control y monitoreo ambiental, teniendo en cuenta la dimensión técnica, social, ambiental, reglamentaria y económica, para tomar decisiones en el marco de la ecoeficiencia para el desarrollo del país.

Se deben analizar alternativas de optimización minera, portuaria y energética de los fenómenos, equipos y procesos relacionados con la cadena de estos tres sectores, incluyendo métodos y tecnologías que incrementen su eficiencia, la aplicación de tecnologías más limpias y no convencionales y el análisis de los impactos ambientales. 


\section{REFERENCIAS BIBLIOGRÁFICAS}

Haeckel Ernest, El Origen de la Vida. Universidad de Berlín 1886

Hernández Luis Carlos, Impactos Ambientales en la Minería a Cielo Abierto. Ecotierra 2010

Holdrigge Leslie R, Ecología basada en zonas de vida, Instituto de cooperación para la agricultura, San José, Costa Rica 2000. 159p.

Ministerio de Ambiente, Vivienda y Desarrollo Territorial y Ministerio de Minas y Energía. Guía Metodológica para Evaluación de Impactos Ambientales por actividades mineras 2009

Ministerio del Medio Ambiente, Guías ambientales para proyectos de generación eléctrica, 1997.

Ministerio del Medio Ambiente. Guía Ambiental para Sector Portuario 2000.

Ministerio de Minas y Energía. Propuesta para el Mejoramiento del Recurso Energético en Colombia. 2007 